\title{
FINITE ELEMENT SIMULATION OF THE STEEL PLATES HOT ROLLING PROCESS
}

\author{
Miguel A. Cavaliere, Marcela B. Goldschmit \\ and Eduardo N. Dvorkin \\ Center for Industrial Research, FUDETEC \\ Av. Córdoba 320 \\ 1054, Buenos Aires, Argentina
}

February 15, 2001

\begin{abstract}
In this paper we discuss our finite element procedure for simulating the hot rolling of flat steel products. We couple an Eulerian rigid-viscoplastic model of the steel plates deformation to a Lagrangian elastic model of the rolls deformation. This latter model incorporates the bending deformation of the work rolls supported by the back-up rolls and the flattening of the contact areas (Hertz problem) via an enhanced beam model.

The finite element model is validated comparing its predictions with actual industrial measurements and then it is used to analyze different rolling set-ups.

Key Words: metal forming; hot strip rolling; finite elements; thermal crown; roll bending; roll flattening
\end{abstract}

\section{Introduction}

In previous publications [1] to [6] we presented an Eulerian formulation for modeling metal forming processes which is based on the flow formulation (rigid - viscoplastic material models) [7] implemented via Thompson's pseudo - concentrations technique [8][9]. When developing a 3D finite element model, for simulating the hot rolling of steel plates, it is necessary to include in this model the rolls deformation, because it plays a central role in determining the resulting profile and flatness ${ }^{1}$ of the rolled plates [10]. It can be safely assumed that the rolls (work and back-up rolls as per Fig. 1) deform within the elastic range; hence, it is necessary to couple the Eulerian rigid - viscoplastic model of the rolled steel plates deformation to an elastic Lagrangian model of the rolls deformation.

\footnotetext{
${ }^{1}$ The concepts of plate profile and flatness are going to be discussed in Appendix A
} 
In the second section of this paper we summarize the relevant aspects of our Eulerian rigid - viscoplastic formulation and of its coupling to the elastic Lagrangian model that describes the rolls deformation under the loading indicated in Fig. $2\left(F_{1}\right.$ and $F_{2}$ are forces introduced by an hydraulic rolls bending system; this device is used to control the plates profile and flatness [10]).

When modeling the rolls deformation we assume a linear elastic material behavior; however, we consider several geometrical nonlinearities : contact between the work and back-up rolls and flattening of the contact areas (Hertz problem). The simulation of these phenomena is achieved using an enhanced beam model rather than a more expensive 3D model of the rolls.

In the third section of this paper we present a detailed description of the nonlinear enhanced beam model that we developed for simulating the rolls deformation.

With the coupled formulation implemented in our finite element code METFOR we develop, in the fourth section of this paper, several industrial applications.

\section{The coupled Eulerian - Lagrangian formula- tion}

In this section we briefly describe the basis of our simulation procedure:

- The Eulerian formulation that models the rolled plates deformation using the flow formulation implemented via Thompson's pseudo - concentrations technique.

- The coupling of that Eulerian formulation to the Lagrangian formulation that models the rolls deformation (to be developed in the next section)

\subsection{The Eulerian formulation}

We use a fixed mesh with the material moving inside the mesh; at each point interior to the mesh we define a variable named pseudo - concentration $(c)$ :

$c \geqslant 0 \Longleftrightarrow$ there is material at the point,

$c<0 \Longleftrightarrow$ there is no material at the point.

Being $(\underline{\dot{u}})$ the velocity field inside the mesh, the $c$-distribution fulfils the following equations: 


$$
\begin{aligned}
& \underline{\dot{u}} \cdot \underline{\nabla} c=0 \quad \text { (stationary problems) } \\
& \left.\frac{\partial c}{\partial t}+\underline{\dot{u}} \cdot \underline{\nabla} c=0 \quad \text { (transient problems }\right)
\end{aligned}
$$

For modeling the rolled steel behavior we use a rigid - viscoplastic constitutive relation [11]; the material flow is described via a viscoplastic associated von Mises model. Hence, the material flow is incompressible.

We impose the incompressibility constraint via an augmented Lagrangian procedure [12] to [17]. Equilibrium is reached via an iterative procedure because:

- The material constitutive relation is nonlinear.

- The augmented Lagrangian technique iteratively builds the pressure field.

We use a modified Uzawa's algorithm [14] and in the same iterative loop we solve the nonlinearities coming from the constitutive model and make the augmentation procedure.

The equations for the $\mathrm{k}$-th equilibrium iteration, obtained using the Principle of Virtual Work and the augmented Lagrangian technique are [2]:

$$
\begin{gathered}
\int_{V} 2 \mu^{(k-1)} \Delta \dot{\varepsilon}_{i j}^{\prime} \delta \Delta \dot{\varepsilon}_{i j}^{\prime} d v+\int_{V} \varkappa \Delta \dot{\varepsilon}_{v} \delta \Delta \dot{\varepsilon}_{v} d v= \\
\int_{V} f_{i}^{v} \delta \Delta \dot{u}_{i} d v+\int_{S_{\sigma}} t_{i} \delta \Delta \dot{u}_{i} d v- \\
\int_{V} s_{i j}^{(k-1)} \delta \Delta \dot{\varepsilon}_{i j}^{\prime} d v-\int_{V}\left(p^{(k-1)}+\varkappa \dot{\varepsilon}_{v}^{(k-1)}\right) \delta \Delta \dot{\varepsilon}_{v} d v
\end{gathered}
$$

and,

$$
\begin{aligned}
& \dot{u}_{i}^{(k)}=\dot{u}_{i}^{(k-1)}+\Delta \dot{u}_{i}^{(k)} \\
& \dot{\varepsilon}_{i j}^{\prime(k)}=\dot{\varepsilon}_{i j}^{\prime(k-1)}+\Delta \dot{\varepsilon}_{i j}^{\prime(k)} \\
& \dot{\varepsilon}_{v}^{(k)}=\dot{\varepsilon}_{v}^{(k-1)}+\Delta \dot{\varepsilon}_{v}^{(k)} \\
& p^{(k)}=p^{(k-1)}+\varkappa \dot{\varepsilon}_{v}^{(k)}
\end{aligned}
$$

In the above equations,

$s_{i j}:$ deviatoric stress components, 
$p$ : hydrostatic stress component,

$\dot{\varepsilon}_{i j}^{\prime}$ : deviatoric strain rate components (viscoplastic strain rate tensor),

$\dot{\varepsilon}_{v}$ : volumetric strain rate component (it is constrained to be zero),

$\varkappa$ : penalty parameter; the advantage of the augmented Lagrangian procedure over a standard penalty procedure is the possibility of using smaller values for this parameter and therefore the possibility of working with better conditioned matrices. In our Ref. [2] we presented several numerical examples in which we showed the better performance of the augmented Lagrangian procedure as compared with the standard penalty procedure,

$\mu$ : viscosity, derived using the constitutive viscoplastic relation [7]. For the special case of a rigid - plastic material we get,

$$
\mu=\frac{\sigma_{y}(\bar{\varepsilon})}{3 \dot{\bar{\varepsilon}}}
$$

$\dot{\bar{\varepsilon}}$ : material derivative of the equivalent plastic strain.

$$
\begin{gathered}
\dot{\bar{\varepsilon}}=\left(\frac{2}{3} \dot{\varepsilon}_{i j} \dot{\varepsilon}_{i j}\right)^{\frac{1}{2}} \\
\dot{\bar{\varepsilon}}=\frac{D \bar{\varepsilon}}{D t}=\frac{\partial \bar{\varepsilon}}{\partial t}+\underline{\dot{u}} \cdot \underline{\nabla} \bar{\varepsilon}
\end{gathered}
$$

$f_{i}^{v}$ : external forces acting on the body, per unit volume of the spatial configuration,

$t_{i}$ : external surface forces acting on the body,

$V$ : volume of the body spatial configuration $(c>0)$. However, in order to be able to integrate over the complete mesh, at those points where $c<0$ we use $\mu=10^{-3} \mu_{\text {actual material }}$,

$S_{\sigma}$ : external surface where loads are prescribed.

In Eqn.(3d) we iteratively build the pressure field using the augmented Lagrangian technique.

For discretizing the equilibrium equations (2) we use finite elements based on the method of mixed interpolation of tensorial components. For 2D problems we use the $Q M I T C-3 F$ quadrilateral element, which was described in our Ref. $[2]$ and for $3 \mathrm{D}$ problems we use the (H1-P0)-3F element described in our Ref. [6]. Both element formulations fulfil the following requirements: 
- They satisfy Irons' Patch Test.

- They do not contain spurious zero energy modes.

- They do not lock due to the incompressibility constraint.

For discretizing the transport equations (1a or $1 \mathrm{~b}$ ) we use standard isoparametric elements: quadrilateral $\left(Q_{1}\right)$ elements for the $2 \mathrm{D}$ problems and hexahedral $\left(H_{1}\right)$ elements for the 3D problems. In all cases, for solving the advective transport equations we use the SUPG technique [17] [18].

The equivalent plastic strains are obtained integrating the following equations,

$$
\begin{aligned}
\underline{\dot{u}} \cdot \underline{\nabla} \bar{\varepsilon} & =\frac{\langle c\rangle}{|c|} \dot{\bar{\varepsilon}} \text { (stationary problems) } \\
\frac{\partial \bar{\varepsilon}}{\partial t}+\underline{\dot{u}} \cdot \underline{\nabla} \bar{\varepsilon} & =\frac{\langle c\rangle}{|c|} \dot{\bar{\varepsilon}} \text { (transient problems) }
\end{aligned}
$$

Some notes regarding our Eulerian formulation:

- The material moves inside a fixed mesh.

- It provides the free surfaces in stationary and transient problems without any special free surface algorithm.

- It does not require a remeshing algorithm, usually needed when using Lagrangian or Eulerian - Lagrangian formulations.

The contact problem between the blank and the tools (plate and work rolls) is modelled using $c$-dependent boundary conditions [1]; for $\dot{u}_{n}$, the velocity component normal to a tool surface, we use:

$$
\begin{aligned}
& c<0 \Longrightarrow \dot{u}_{n}=\text { free } \\
& c \geqslant 0 \Longrightarrow \dot{u}_{n}=0
\end{aligned}
$$

For modeling the friction between the rolls and the steel plates we use either a constant friction law or a Coulomb friction law [19]. It is important to notice that the location of the non-slip points is an analysis result [5]. 


\subsection{Staggered iterative solution}

The coupling between the Eulerian model that we described in the previous subsection and the Lagrangian rolls deformation model that we are going to describe in the following section is performed via an iterative staggered scheme that is composed by:

- An OUTER LOOP that incorporates the rolls deformation model.

- An INNER LOOP or equilibrium loop.

We describe the staggered iterative scheme in what follows:

\section{OUTER LOOP}

1. $k=0$

2. Assume for the rolls a trial deformation $\longrightarrow\left(\underline{U}_{\text {rolls }}^{(k)}\right)$

3. $k=k+1$

4. Keep fixed $\left(\underline{U}_{\text {rolls }}^{(k-1)}\right)$ and solve the equilibrium equations (either stationary flow or transient flow, in the latter case solve for the step $t \longrightarrow t+\Delta t$ ) GO TO THE INNER LOOP

5. From the above loop get the loads imposed by the rolled plates on the rolls $\left(\underline{R}^{(k)}\right)$

6. Solve the enhanced beam model under the loads $\underline{R}^{(k)}$ and the bending forces $\longrightarrow\left(\underline{U}_{\text {rolls }}^{(k)}\right)$

7. IF $\frac{\left\|\underline{U}_{\text {rolls }}^{(k)}-\underline{U}_{\text {rolls }}^{(k-1)}\right\|_{2}}{\left\|\underline{U}_{\text {rolls }}^{(k)}\right\|_{2}} \leqslant U T O L$

THEN $\longrightarrow C O N V E R G E N C E$

ELSE $\longrightarrow$ GOTO 3 
INNER LOOP (EQUILIBRIUM LOOP)

- For stationary problems start from a trial $c$-distribution and zero velocities $\underline{\dot{u}}^{(0)}=0$.

- For transient problems, $t \longrightarrow t+\Delta t$, start from the converged solution at time $t$.

- Keep constant the rolls deformations (point 4 in the outer loop).

1. $l=0$

2. $l=l+1$

2.a. $r=0 ; \underline{\dot{u}}^{(r)}=\underline{\dot{u}}^{(l-1)}$

2.b. $r=r+1$

Solve Eqns. (2 and $3 a$ to 3d) keeping constant the $c$-distribution and the $\bar{\varepsilon}$-distribution.

2.c. IF $\frac{\left\|\underline{\dot{u}}^{(r)}-\dot{\underline{u}}^{(r-1)}\right\|_{2}}{\left\|\underline{\underline{\dot{u}}}^{(r)}\right\|_{2}} \leqslant \dot{U} T O L$. AND. $\left\|\dot{\varepsilon}_{v}\right\|_{\infty} \leqslant \dot{V} T O L$

THEN $\longrightarrow \underline{\dot{u}}^{(l)}=\underline{\dot{u}}^{(r)}$ GO TO 3

ELSE GO TO $2 . b$

3. Calculate the $c$-distribution and the $\bar{\varepsilon}$-distribution using Eqns. (1a or $1 b$ and $7 a$ or $7 b$ )

4. $I F l=1$

\section{THEN GO TO 2}

ELSE

$I F \frac{\left\|\underline{\dot{u}}^{(l)}-\dot{\underline{\dot{u}}}^{(l-1)}\right\|_{2}}{\left\|\underline{\dot{u}}^{(l)}\right\|_{2}} \leqslant \dot{U} T O L$

$T H E N \longrightarrow C O N V E R G E N C E$

ELSE $\longrightarrow G O T O 2$ 


\section{Simulation of the rolls deformation. The en- hanced beam model}

In this section we present the enhanced beam model that we developed to simulate the rolls elastic deformation.

\subsection{Contact between work and back-up rolls. The Hertz elements}

We discretize the work roll and its corresponding back-up roll using Hermitian beam elements that include the shear deformation [20]. In the area where contact between both rolls is possible we define pairs of matching nodes (one on each beam model) and between them we implement a "node-to-node" contact algorithm (usually the rolls are parallel and the matching nodes are located at the intersections between the beam axes and a common normal). We interpose between the matching nodes an ad hoc element that models the flattening of both surfaces in contact: our new Hertz element.

For the $i$-th Hertz element connecting the matching nodes $N_{i}^{w}$ (corresponding to the work roll) and $N_{i}^{b}$ (corresponding to the back-up roll), using the nomenclature in Fig. 3, we define the initial gap,

$$
\delta_{i}^{0}=\left|\underline{r}_{N_{i}^{w}}-\underline{r}_{N_{i}^{b}}\right|-\left(R_{i}^{w}+R_{i}^{b}\right)
$$

please notice that due to the rolls mechanical and thermal crown the radius $\left(R_{i}^{w}\right.$ and $R_{i}^{b}$ ) are variable node to node.

Loading the beam system (work + back-up rolls) with the forces determined in the Eulerian model plus the bending forces, we get the nodal displacements $\underline{U}_{N_{i}^{w}}$ and $\underline{U}_{N_{i}^{b}}$; hence, the gap undergoes a change, from $\delta_{i}^{0}$ to $\left(\delta_{i}^{0}+\Delta_{U} \delta_{i}\right)$,

$$
\Delta_{U} \delta_{i}=\left(\underline{U}_{N_{i}^{b}}-\underline{U}_{N_{i}^{w}}\right) \cdot \underline{r}_{i}=u_{i}^{b}-u_{i}^{w}
$$

where,

$$
\underline{r_{i}}=\frac{\underline{r}_{N_{i}^{b}}-\underline{r}_{N_{i}^{w}}}{\left|\underline{r}_{N_{i}^{b}}-\underline{r}_{N_{i}^{w}}\right|} .
$$

The gap is also affected by the Poisson effect in the bent beams [21],

$$
\begin{aligned}
\Delta_{\nu} \delta_{i} & =\left(\underline{U}_{N_{i}^{b}}^{v}-\underline{U}_{N_{i}^{w}}^{\nu}\right) \cdot \underline{r_{i}}=u_{i}^{\nu^{b}}-u_{i}^{\nu^{w}} \\
\left|\underline{U}_{N_{i}^{k}}^{v}\right| & =\frac{\nu^{k}\left|\underline{M}_{i}^{k}\right|\left(R_{i}^{k}\right)^{2}}{2 E^{k} I_{i}^{k}}
\end{aligned}
$$


the second equation was obtained from Ref. [22] and both displacements act in the direction of the bending displacements but with opposite sense. In the above equation,

$E^{k}, \nu^{k} \quad$ : Yong's modulus and Poisson ratio for the work roll $(k=w)$ or for the back-up roll $(k=b)$,

$\underline{M}_{i}^{k}, I_{i}^{k}$ : bending moment and inertia moment for the work roll/back-up roll section related to the $i$-th Hertz element.

Therefore, the rolls bending makes the initial gap evolve from $\delta_{i}^{0}$ to the value,

$$
\delta_{i}^{1}=\delta_{i}^{0}+\Delta_{U} \delta_{i}+\Delta_{\nu} \delta_{i}
$$

When $\delta_{i}^{1}>0$ the nodes $N_{i}^{w}$ and $N_{i}^{b}$ are not in contact and when $\delta_{i}^{1}<0$ both nodes are in contact and there is a contact force $\left(P_{i}\right)$ among them. Being $D$ the axial distance between equally spaced nodes on the roll axes and $b_{i}$ the width of the contact zone for the $N_{i}^{w}-N_{i}^{b}$ Hertz element, we can write [23]

$$
\begin{aligned}
b_{i} & =\sqrt{\frac{2 P_{i} K_{R_{i}} C_{E}}{\pi D}} \\
K_{R_{i}} & =\frac{2 R_{i}^{w} R_{i}^{b}}{R_{i}^{w}+R_{i}^{b}} \\
C_{E} & =\frac{1-\nu^{w}}{E^{w}}+\frac{1-\nu^{b}}{E^{b}}
\end{aligned}
$$

We approximate the radial displacement of each roll surface (flattening) with the superposition of the elastic solutions of a semi-infinite solid $(z \geqslant 0)$ loaded with distributed loads, $p_{n}$, acting on the area $S_{n}=D b_{n}$ in the plane $z=0$. Using a Cartesian coordinate system centered in the area $S_{n}$ the solution of the Boussinesq problem is [24],

$$
\begin{aligned}
w_{i}^{k}\left(x_{i}, y_{i}, z_{i}\right) & =\sum_{n=1}^{N_{\text {Hert } z}} \frac{\left(1+\nu^{k}\right)}{2 E^{k} \pi} \iint_{S_{n}} p_{n}\left(x \prime, y^{\prime}\right)\left[\frac{2\left(1-\nu^{k}\right)}{r}+\frac{z_{i}^{2}}{r^{3}}\right] d x \prime d y \prime \\
r & =\sqrt{\left(x_{i}-x \prime\right)^{2}+\left(y_{i}-y^{\prime}\right)^{2}+z_{i}^{2}} \\
p_{n} & =\frac{P_{n}}{S_{n}}
\end{aligned}
$$

where $N_{\text {Hertz }}$ is the number of Hertz elements. 
With a close form solution we can calculate the integral on the r.h.s. of Eqn. (14a). The contribution of each roll $(k=w$ or $k=b)$ to the flattening of the i-th Hertz element is,

$$
a_{i}^{k}=w_{i}^{k}(0,0,0)-w_{i}^{k}(0,0, R) .
$$

The flattening of the i-th Hertz element is the addition of all the contributions we evaluated above; hence, the gap evolves from its original value to a final value,

$$
\delta_{i}=\delta_{i}^{0}+\Delta_{U} \delta_{i}+\Delta_{\nu} \delta_{i}+a_{i 1}+a_{i 2}=\delta_{i}^{1}+a_{i 1}+a_{i 2}
$$

the contact condition imposes,

$$
\delta_{i}=0
$$

For solving the nonlinear beam system we implemented the following algorithm:

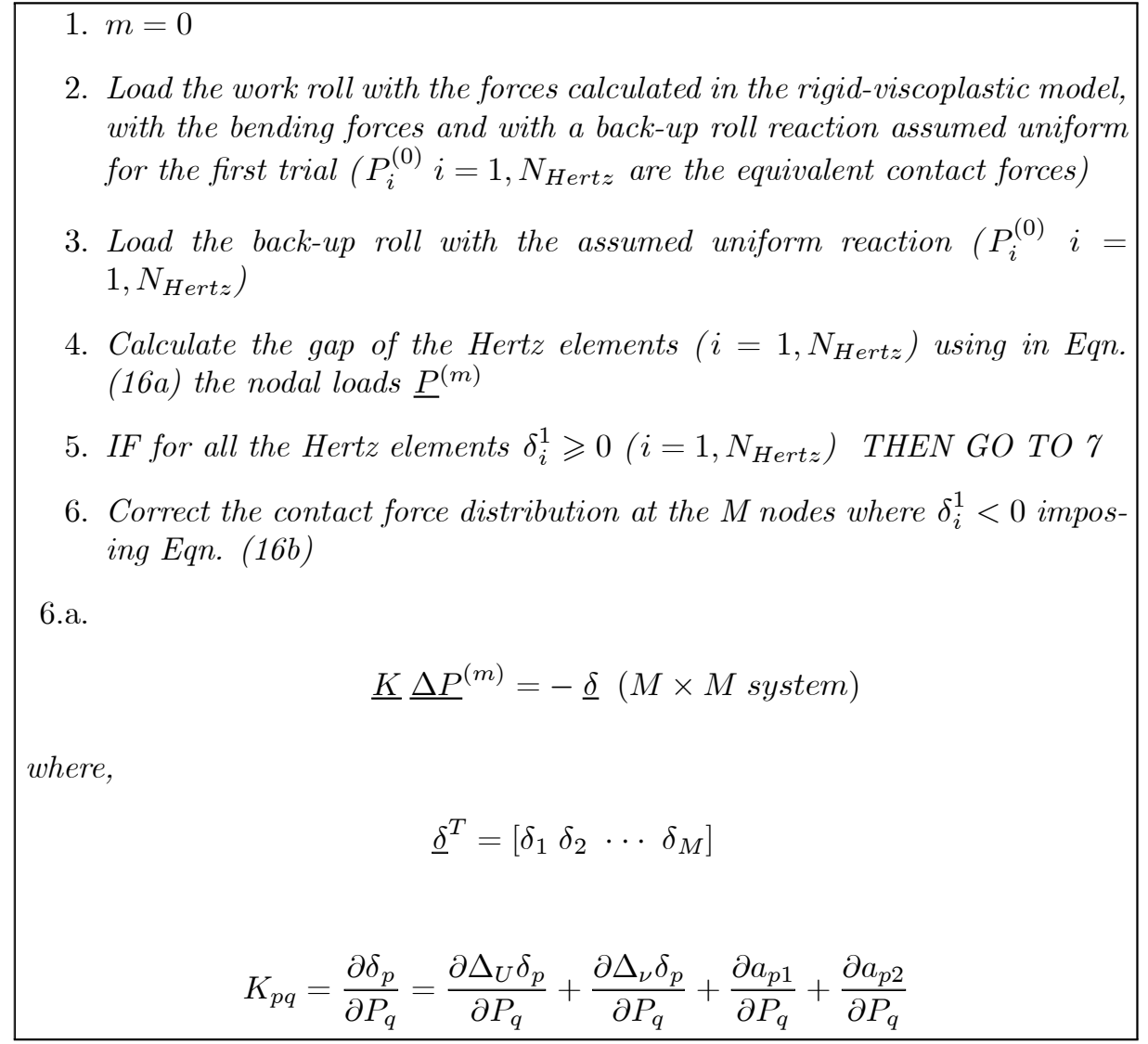




\begin{tabular}{l} 
6.b. $m=m+1$ \\
6.c. $\underline{P}^{(m)}=\underline{P}^{(m-1)}+\underline{\Delta P^{(m)}}$ \\
6.d. IF $P_{i}^{(m)}<0$ THEN $P_{i}^{(m)}=0 i=1, M$ \\
6.e. $I F \frac{\left\|\underline{P}^{(m)}-\underline{P}^{(m-1)}\right\|_{2}}{\left\|\underline{P}^{(m)}\right\|_{2}} \leqslant$ PTOL THEN GOTO 7 \\
ELSE GOTO 4 \\
7. CONVERGENCE \\
\hline
\end{tabular}

\subsection{Contact between work rolls and the rolled plates}

In order to determine the total rolls deformation it is necessary to add, to the deformation calculated as detailed above, the flattening of the work rolls due to their contact with the rolled steel plates. For this purpose we use Eqn. (14a) integrated over the contact surface between the work roll and the rolled steel plate.

\section{Industrial applications of the model}

In this section we present several simulations developed, for the hot rolling mill in Fig. 1, using our modeling procedure. Our objectives are to qualify, for roughing and finishing stands, the developed finite element technique and to demonstrate its potential for predicting, in actual engineering applications, the plate profiles that can be expected when using in the rolling mill different set-up alternatives. The analyses that we describe in what follows need to render very accurate results because, in the modern industrial practice, the boundary between acceptable plate profiles and non-acceptable ones is defined by profile differences from two to three orders of magnitude smaller than the plates thickness.

As it is well known, the solution of the transport equation (1a) or (1b), that defines the plates profile, usually does not provide the needed accuracy due to numerical diffusions that can be minimized, using the available numerical techniques[17][18], but not completely eliminated, specially in the case of non regular meshes. However, in our numerical experimentation we found that this lack of accuracy in determining the position of the surfaces $c=0$, that define the plates geometry, does not have an important influence when calculating the separating forces; hence, the most accurate procedure for determining the rolled plates profile is by considering the model predictions for the work rolls deformation. 


\subsection{Validation of the model for a roughing stand (R4)}

In our previous references [3],[4] and [6] we publised the result of a set of industrial tests that were developed for validating our finite element model when applied to a roughing stand. The industrial tests were carried out on the roughing stand R4 and the obtained results are reproduced in Fig. 4. The larger difference between the finite element predicted plate profile at the stand exit and the measured plate profile at that point is located at approx. $\pm 450 \mathrm{~mm}$ from the stand center. This is because in our analysis, we did not incorporate into the model geometry the rolls wear, which can be characterized with the typical R4 roll wear profile shown in Fig.5.

The obtained results are quite satisfactory and therefore we can consider that METFOR is a valid tool for modeling a roughing stand.

\subsection{Parametric analyses on a roughing stand ( $\mathrm{R} 4)$}

The purpose of these analyses is to investigate the effects, on the plate profile produced at this roughing stand, of:

- The plate profile at the stand entrance (that is to say the plate profile produced by the upstream stand R3)

- The total crown of the work rolls ${ }^{2}$.

For our analyses we considered the following stand data:

\begin{tabular}{|c|c|c|}
\hline & Back-up rolls & Work rolls \\
\hline Material & steel & casted iron \\
\hline E $[\mathrm{Gpa}]$ & 205.8 & 172.5 \\
\hline Central diameter $[\mathrm{mm}]$ & 1185.86 & 622.94 \\
\hline Total crown $[\mathrm{mm}]$ & 0.00 & see Table III \\
\hline Peripherical speed $[\mathrm{m} / \mathrm{s}]$ & & 2.136 \\
\hline
\end{tabular}

Table I. Rolls data

\begin{tabular}{|c|c|}
\hline Temperaure $\left[{ }^{\circ} \mathrm{C}\right]^{3}$ & 1100 \\
\hline Yield stress $[\mathrm{MPa}]^{4}$ & 117.6 \\
\hline Width $[\mathrm{mm}]$ & 1000 \\
\hline Upstream plate crown $[\mathrm{mm}]$ & see Table III \\
\hline Upstream plate thickness $[\mathrm{mm}]$ & 54 \\
\hline Downstream plate thickness $[\mathrm{mm}]$ & 30 \\
\hline
\end{tabular}

Table II. Plate data

\footnotetext{
${ }^{2}$ The concept of roll crown is going to be discussed in Appendix A.

${ }^{3}$ Assumed constant through the plate thickness and through the plate width. This approximation is going to be improved with a coupled thermo - mechanical model.

${ }^{4}$ Assumed constant and calculated using the above defined temperature [26]. This approximation is going to be improved with a coupled thermo - mechanical - metallurgical model.
} 
In our parametric analysis we considered the following cases:

\begin{tabular}{|c|c|c|}
\hline Case & Total crown of the work rolls $[\mathrm{mm}]$ & Upstream plate crown $[\mathrm{mm}]$ \\
\hline $\mathbf{1}$ & +0.1 & 0.0 \\
\hline $\mathbf{2}$ & +0.1 & +0.4 \\
\hline $\mathbf{3}$ & +0.1 & -0.4 \\
\hline $\mathbf{4}$ & -0.1 & 0.0 \\
\hline $\mathbf{5}$ & -0.1 & +0.4 \\
\hline $\mathbf{6}$ & -0.1 & -0.4 \\
\hline
\end{tabular}

Table III. Parametric analysis

In all cases we considered a constant friction law with $m=0.7$.

In Fig. 6 we present for the six analyzed cases the "plate - work rolls" separating force distribution.

From the results plotted above we conclude that:

- The separating force distribution is not dependent on the plate profile upstream the stand R4.

- Since the roll deformation (bending and flattening) is determined by the separating forces, and the plate profile downstream the stand R4 is determined by the roll deformation, we can conclude that for this stand the produced plate profile is not dependent on the incoming plate profile.

\subsection{Validation of the model for a finishing stand (F10)}

In this subsection we present the qualification of our finite element model for the analysis of a finishing stand, comparing its predictions with actual industrial measurements performed on the rolling mill.

It is very important to include in the analysis an accurate description of the rolls thermal crown ${ }^{5}$; hence, to analyze the rolls thermal evolution and the corresponding thermal crown evolution we developed the finite element system TCROWN, described elsewhere [25], composed of two modules:

- ROLLTEM: predicts the work rolls thermal evolution. It is a 2D axisymmetric finite element model. Even tough the axisymmetric model cannot predict the temperature peaks on the roll surface, these peaks have a very low thickness penetration [10] and can be neglected for determining the rolls thermal expansion. A.

${ }^{5}$ The concepts of thermal and mechanical rolls crown are going to be discussed in Appendix 
- ROLLEXP: calculates, using a thermo - elastic model, the work rolls thermal crown evolution.

Using TCROWN, and measurements of the rolls surface temperature performed after the rolling schedule described in Fig. 7, we characterized the work rolls thermal evolution as shown in the same figure.

In Fig. 8 we describe the temperature distribution corresponding to the instants at which the last rolled coil exits the F10 stand. In Fig. 9 we plot the roll profile developed by the temperature distribution described in the previous figure and the initial cold roll profile, from them the thermal radial expansion and the resulting thermal crown can be obtained.

To perform our finite element analysis we used the following data:

1. Upstream plate profile with a relative crown (crown/thickness $)^{6}$ equal to the relative crown measured on the downstream plate profile.

2. Plate constant yield stress (200.9 MPa) calculated matching the measured total separating force $(8124.2 \mathrm{kN})$ with the numerically predicted total separating force.

3. Bending force $F_{1}=156.8 k N ; F_{2}=0$ (see Fig. 2).

In Fig. 10 we compare the numerical plate profile predicted by METFOR, using the above data, with the distribution of on-line measurements performed on the same plate by the thickness measuring equipment of the rolling mill.

If instead of calculating the yield stress as described above, we use the expressions in [26] with a stand temperature of $920{ }^{\circ} \mathrm{C}$ we obtain a total separating force $25 \%$ higher than the actual one; even tough this discrepancy is of the order of the discrepancies reported in [27] to [29] for models that do not include the thermo - mechanical - metallurgical coupling, it is too high for the purpose of predicting the plate profile. Of course, when we implement a coupled thermo - mechanical - metallurgical model we will not need to go through the measurement and matching of the separating forces.

The obtained results are quite satisfactory and therefore we can consider that METFOR is also a valid tool for modeling a finishing stand.

\footnotetext{
${ }^{6}$ The influence of the relative crown on the plate flatness is going to be discussed in Appendix A.
} 


\subsection{Parametric analyses on a finishing stand (F10)}

In this subsection we analyze the plate profile produced in the last finishing stand when two different strategies are used for cooling the work rolls; in Fig. 11 we represent, for each of the two water cooling configurations that we are going to consider, its heat transfer capacity distribution.

Using the finite element system TCROWN we evaluate the work roll temperature distributions (Fig. 12) and the work roll profiles (Fig. 13) after rolling 14 plates. Using the data in Fig. 13 we analyze the stand with METFOR and obtain the results in Fig. 14.

It is evident that, in the case of a finishing stand, the rolls cooling system has a strong control on the produced plate profiles and therefore on the plate flatness [10].

\section{Conclusions}

We presented a finite element formulation for modeling the hot rolling of steel plates; the formulation couples an Eulerian description of the rolled plates deformation and a Lagrangian description of the rolls deformation.

The Eulerian description of the rolled plates deformation is developed using the classical flow formulation (rigid - viscoplastic material model) implemented via Thompson's pseudo - concentrations technique.

The Lagrangian description of the rolls deformation is developed using a new enhanced beam element; the Hertz element incorporates the bending deformation of the work rolls supported by the back-up rolls and the flattening of the contact areas. It is a nonlinear elastic element and it is much more efficient than the use of $3 \mathrm{D}$ elements.

A future enhancement of the accuracy of our model predictive capability will be achieved by developing a coupled thermo - mechanical - metallurgical model [27] to [30].

Acknowledgement We gratefully acknowledge the support from SIDERAR (San Nicolás, Argentina) for this research. The experimental data reported in this paper was acquired and delivered to us by the process engineers at SIDERAR hot rolling mill. We also acknowledge many discussions on our results, that helped us improve the finite element model 


\section{References}

[1] E.N.Dvorkin and E.G.Petöcz. An effective technique for modelling 2D metal forming processes using an Eulerian formulation. Engineering Computations 1993; 10:323-336.

[2] E.N.Dvorkin, M.A.Cavaliere and M.B.Goldschmit. A three field element via augmented Lagrangian for modelling bulk metal forming processes. Computational Mechanics 1995; 17: 2-9.

[3] E.N.Dvorkin, M.B.Goldschmit, M.A.Cavaliere and P.M.Amenta. On the modelling of bulk metal forming processes. In Proc. Second ECCOMAS (European Community on Computational Methods in Applied Sciences) Conference on Num. Methods in Engrg., John Wiley \& Sons, 1996.

[4] M.A.Cavaliere, M.B.Goldschmit and E.N.Dvorkin. 3D modeling of bulk metal forming processes via the flow formulation and the pseudo - concentrations technique. In Proceedings Fifth Int. Conf. on Computational Plasticity, (Ed. D.R.J.Owen et al), CIMNE, 1997

[5] E.N.Dvorkin, M.B.Goldschmit, M.A.Cavaliere, P.M.Amenta, O.Marini and W.Stroppiana. 2D finite element parametric studies of the flat rolling process. Journal of Materials Processing Technology 1997;68: 99-107.

[6] E.N.Dvorkin, M.A.Cavaliere, M.B.Goldschmit and P.M.Amenta. On the modeling of steel product rolling processes. International Journal of Forming Processes (ESAFORM) 1998; 1:211-242.

[7] O.C.Zienkiewicz, P.C.Jain and E.Oñate. Flow of solids during forming and extrusion: some aspects of numerical solutions. International Journal of Solid and Structures 1977; 14:15-28.

[8] E.Thompson. Use of the pseudo-concentrations to follow creeping viscous flows during transient analysis. International Journal of Numerical Methods in Fluids 1986; 6:749-761.

[9] E.Thompson and R.E.Smelser. Transient analysis of forging operations by the pseudo-concentrations method. International Journal of Numerical Methods in Engineering 1988; 25:177-189.

[10] V.Ginzburg. High-Quality Steel Rolling: Theory and Practice, Marcel Decker Inc., 1993.

[11] P.Perzyna. Fundamental problems in viscoplasticity. Advances in Applied Mechanics 1996, 9, Academic Press.

[12] C.A.Felippa. Iterative procedures for improving penalty function solutions of algebraic systems. International Journal of Numerical Methods in Engineering 1978; 12:821-836. 
[13] O.C.Zienkiewicz, J.P.Vilotte, S.Toyoshima and S.Nakazawa. Iterative method for constrained and mixed approximation. An inexpensive improvement of FEM performance. Computer Methods in Applied Mechanics and .Engineering 1985; 51:3-29.

[14] M.Fortin and A.Fortin. A generalization of Uzawa's algorithm for the solution of the Navier-Stokes equations. Communications inNumerical Methods in Engineering 1985; 1:205-208.

[15] J.C.Simo and T.Laursen. An augmented Lagrangian treatment of contact problems involving friction. Computers \&5 Structures 1992; 42:97-116.

[16] D.G.Luenberger. Linear and Nonlinear Programming, Addison-Weley, 1984.

[17] O.C.Zienkiewicz and R.L.Taylor, The Finite Element Method (4th. Edition), McGraw-Hill, 1989.

[18] T.J.R.Hughes, The Finite Element Method, Prentice-Hall, 1987.

[19] W.Backofen, Deformation Processing, Addison-Wesley, 1972.

[20] K.J.Bathe, Finite Element Procedures, Prentice Hall, 1996.

[21] A.Hacquin, P.Montmitonnet and J.P.Guillerault. Experimental validation of a rolling stand elastic deformation model. Journal of Materials Processing Technology 1994; 45:199-206.

[22] C.T. Wang, Applied Elasticity, McGraw-Hill,1953.

[23] R.J.Roark and W.C.Young, Formulas for Stress and Strain (5th. Edition), McGraw-Hill, 1975.

[24] A.E.H.Love, A Treatise on the Mathematical Theory of Elasticity, Dover Publications, 1944.

[25] E.N.Dvorkin, M.A. Cavaliere, M.G.Zielonka, M.B. Goldschmit. New developments for the modeling of metal rolling processes. In Proceedings of the European Conference on Computational Mechanics, ECCM: Munich, 1999

[26] M.Pietrzyk and J.Lenard, Thermal-Mechanical Modelling of the Flat Rolling Process, Springer-Verlag, 1991.

[27] C.Devadas, I.V.Samarasekera and E.B.Hawbolt. The thermal and metallurgical state of steel strip during hot rolling: Part I. Characterization of heat transfer. Metallurgical Transactions 1991; 22A:307-319.

[28] C.Devadas, D.Baragar, G.Ruddle, I.V.Samarasekera and E.B.Hawbolt. The thermal and metallurgical state of steel strip during hot rolling: Part II. Factors influencing rolling loads. Metallurgical Transactions 1991; 22A:321-333. 
[29] C.Devadas, I.V.Samarasekera and E.B.Hawbolt. The thermal and metallurgical state of steel strip during hot rolling: Part III. Microstructural evolution. Metallurgical Transactions 1991; 22A:335-349.

[30] M.A. Cavaliere, M.B. Goldschmit, E.N.Dvorkin. On the solution of thermomechanical problems via the pseudo-concentrarions technique. In Proceedings of the European Congress on Computational Methods in Applied Sciences and Engineering, ECCOMAS: Barcelona 2000. 


\section{A Rolling terminology}

In this appendix we are going to briefly comment some concepts pertaining to rolling technology, that have been used in the main body of this paper.

\section{A.1 Plate profile and plate crown}

The transversal section of a rolled steel plate is usually not a rectangle but it has a shape similar to the one schematized in Fig. A.1, this shape is referred to as the plate profile. In order to have a quantitative measure of the difference between the plate thickness at the center of its transversal section and near its edges the plate crown is defined; in the same figure we indicate this definition.

\section{A.2 Plate flatness}

Since the transversal section of a plate is of variable thickness, it is apparent that during rolling different fibers located at different locations across the plate will undergo different elongations; hence, due to the plate continuity, some fibers will be in a tensile state and others in a compressive state. It is well known that the compressed parts may buckle and therefore the plate may loose its flatness.

To quantify the tendency towards buckling at a given stand the following technological parameter is used [10]:

$\delta=\frac{\text { plate crown upstream the stand }}{\text { plate thickness upstream the stand }}-\frac{\text { plate crown downstream the stand }}{\text { plate thickness downstream the stand }}$

The parameter $\delta$ is the difference between the plate relative crowns upstream and downstream the stand. If we neglect the lateral spreading of the plate (a valid assumption for the last finishing stands) then,

- $\delta<0$ indicates a tendency towards buckling at the plate edges,

- $\delta>0$ indicates a tendency towards buckling at the plate center.

Therefore, the objective of a stand set-up is a rolling condition with $\delta=0$ in order to produce a flat plate. However, it has been experimentally defined a range inside which it can be assured the flatness of the plate [10]:

$$
-80\left[\frac{\text { downstream thickness }}{\text { plate width }}\right]^{1.86}<\delta<40\left[\frac{\text { downstream thickness }}{\text { plate width }}\right]^{1.86}
$$




\section{A.3 Rolls profile and crown}

The work rolls and sometimes also the back-up rolls are not straight cylinders, usually the cylinder generatrices have a shape similar to the ones indicated in Fig. A.2 (roll profiles), to compensate the bending of the rolls and therefore produce a plate with a smaller crown. The number used to define a roll profile is the roll crown whose definition is also indicated in the same figure.

As it was discussed in the fourth section the thermal evolution of the work rolls during rolling imposes an evolution of their shape, hence the original or mechanical crown of the rolls is modified by a thermal crown; therefore,

total crown $=$ mechanical crown + thermal crown 


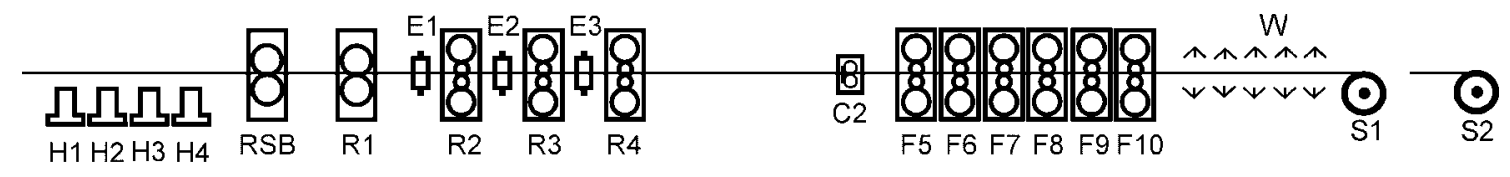

RSB : roughing scale breaker

R1, R2, R3, R4 : roughing stands

E1, E2, E3 : edger rolls

$\mathrm{H} 1, \mathrm{H} 2, \mathrm{H} 3, \mathrm{H} 4$ : re-heating furnaces
C2 : crop shear

F5, F6, F7, F8, F9, F10 : finishing stands

$\mathbf{W}$ : water cooling

$\mathrm{S1}, \mathbf{S 2}$ : sheet coilers

(a) Diagram of a hot rolling mill for steel plates

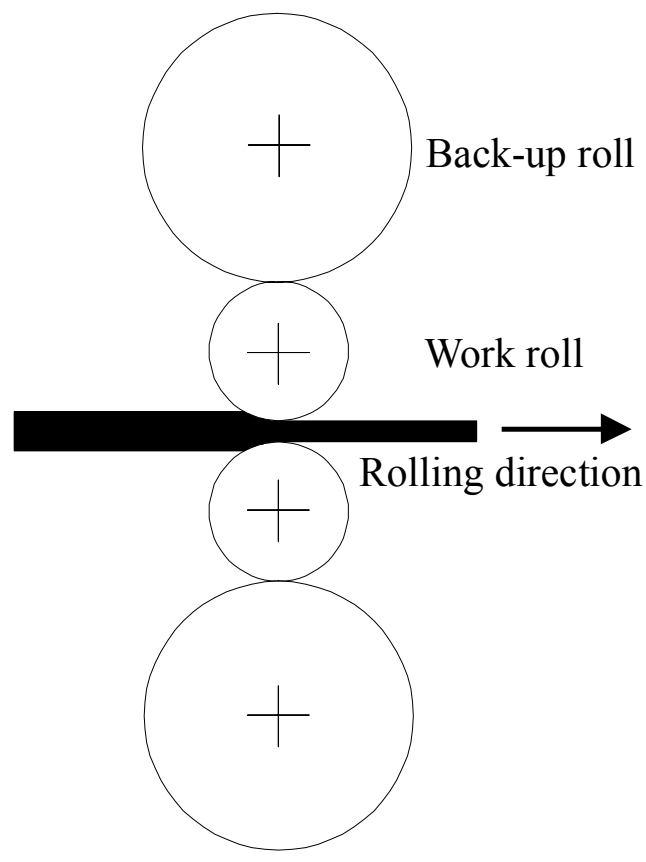

(b) Diagam of a 4-rolls stand (R2 to F10 in the above diagram)

Figure 1. Typical hot rolling mill for steel plates. 


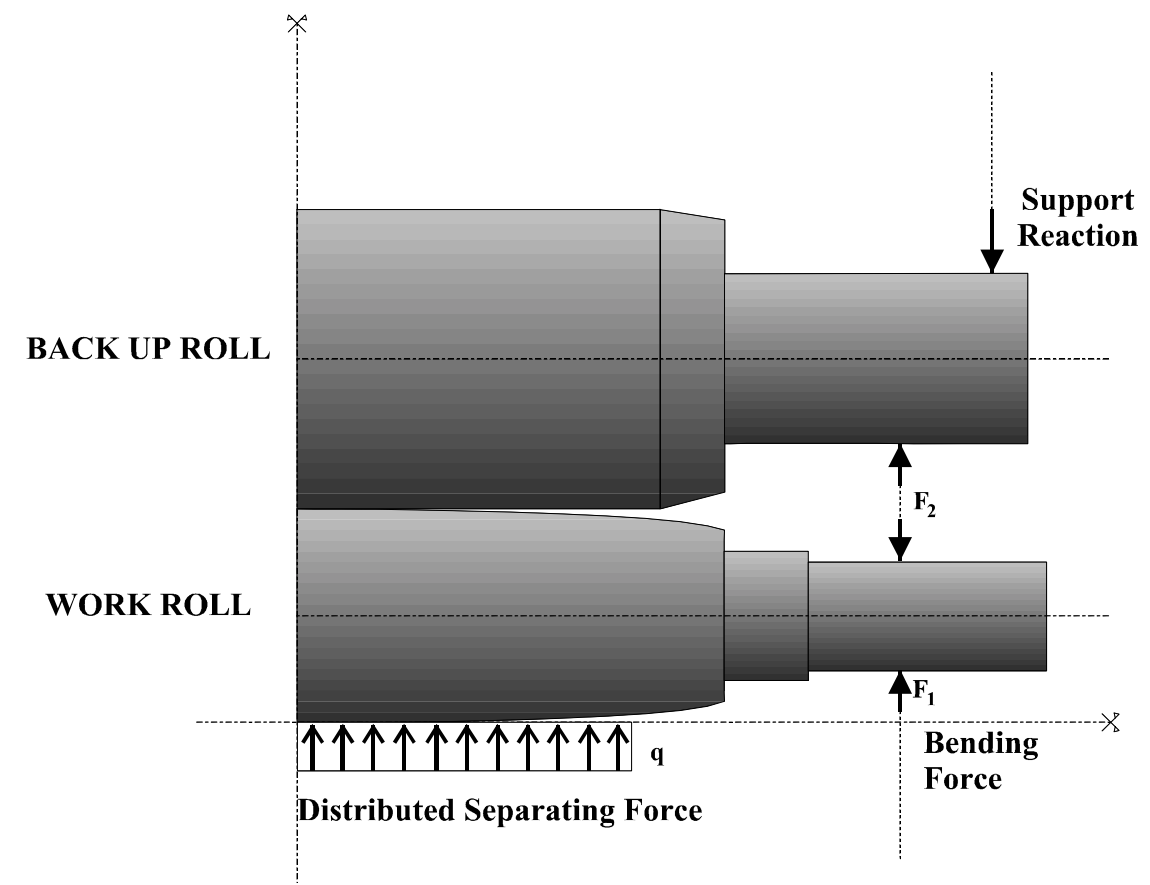

Figure 2. Forces acting on a work/back-up rolls set. 


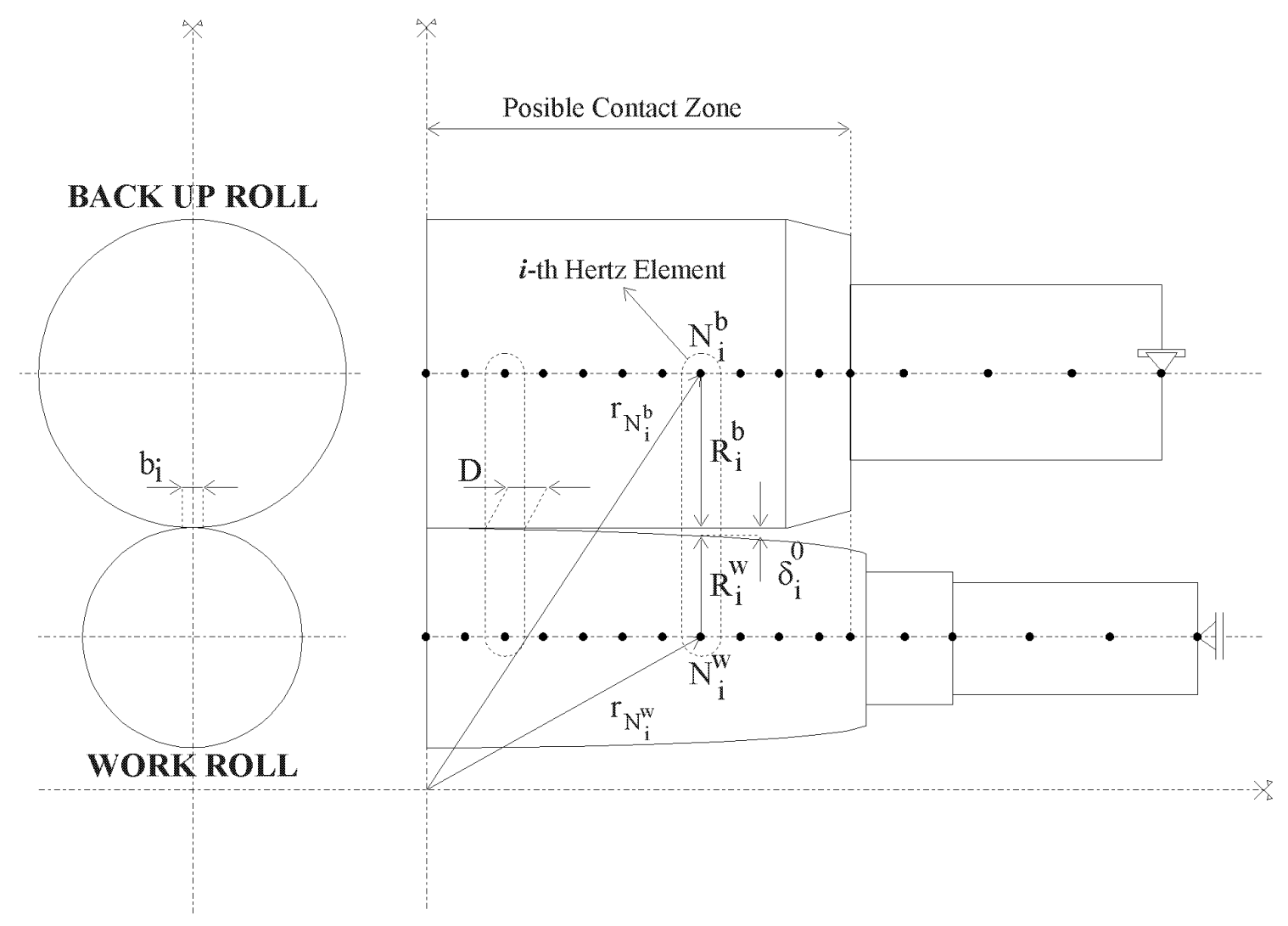

Figure 3. Definition of a Hertz element 


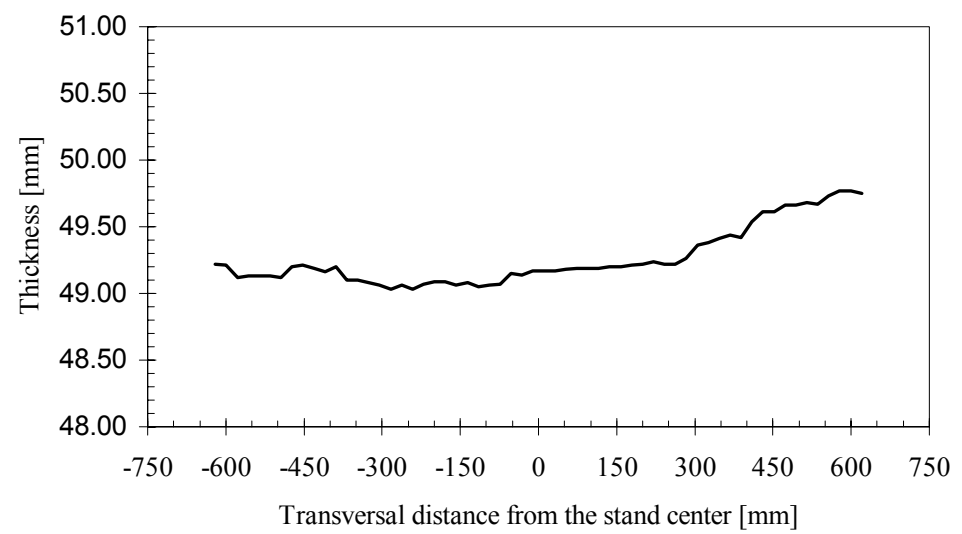

(a) Upstream measured plate profile

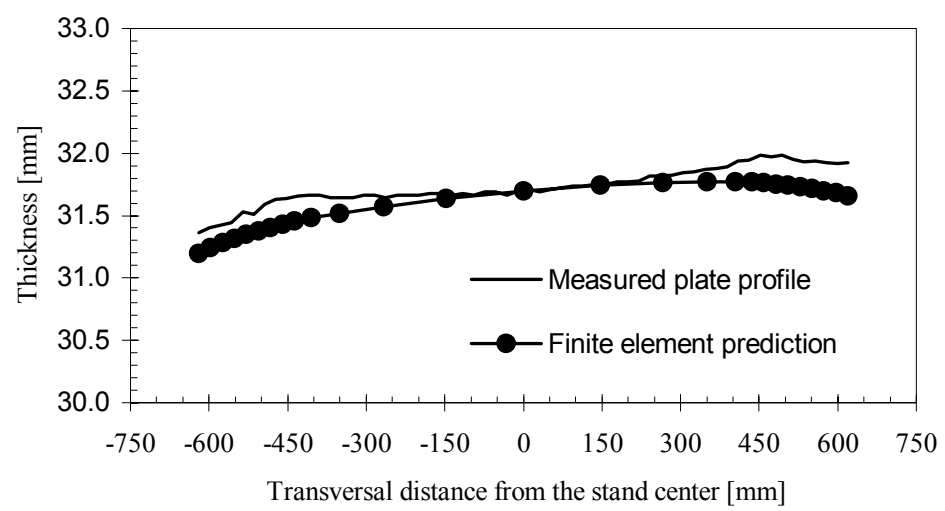

(b) Downstream measured plate profile and finite element prediction

Figure 4. Validation of the model for a roughing stand 


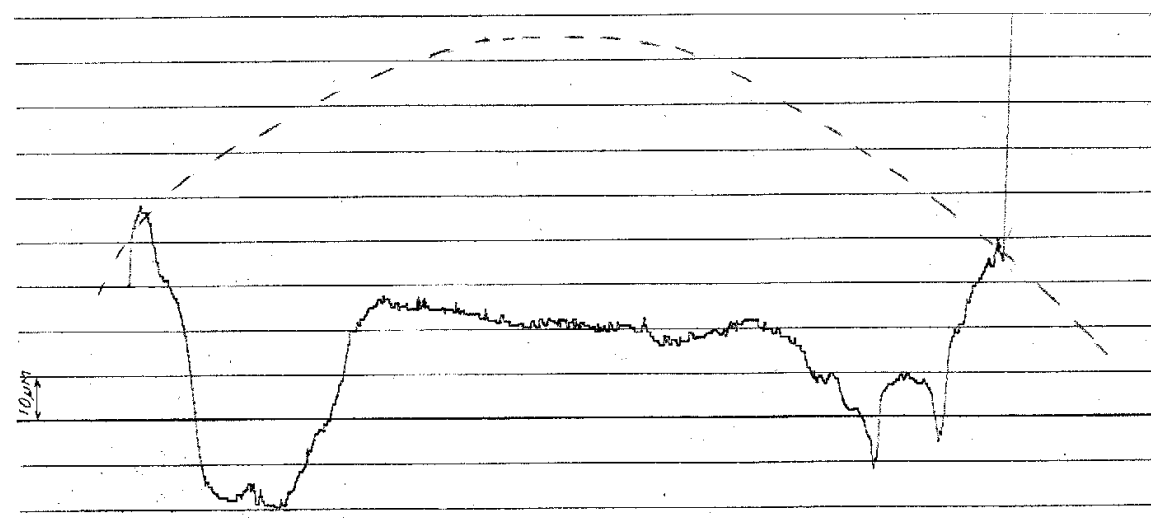

Figure 5. R4 roll wear profile. 


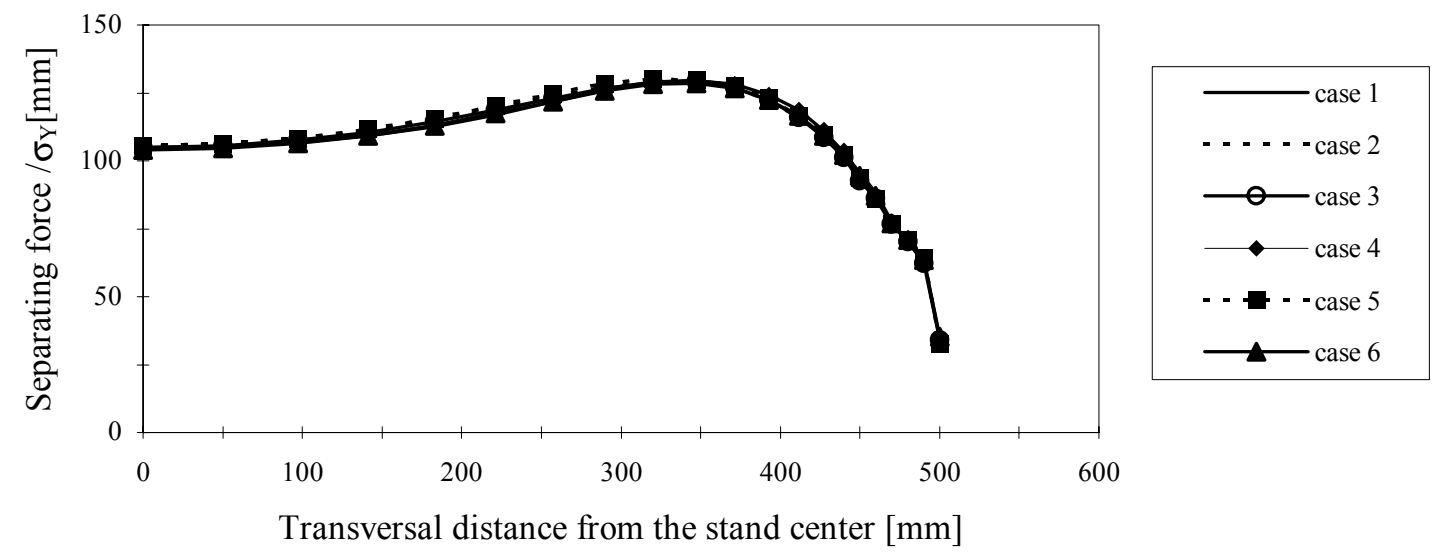

Figure 6. R4 parametric analysis 


\begin{tabular}{|c|c|c|c|c|}
\hline \multicolumn{4}{|c|}{ Stand F10 } & \\
\hline Coil $^{\circ}$ & Width & Time In & Time Out & \\
\hline 1 & 1046 & 0 & 60 & \\
\hline 2 & 1040 & 155 & 215 & \\
\hline 3 & 1042 & 286 & 345 & \\
\hline 4 & 1045 & 373 & 432 & \\
\hline 5 & 1044 & 469 & 531 & \\
\hline 6 & 1041 & 567 & 624 & \\
\hline 7 & 1146 & 653 & 713 & \\
\hline 8 & 1143 & 753 & 813 & \\
\hline 9 & 1257 & 856 & 915 & \\
\hline 10 & 1260 & 962 & 1021 & \\
\hline 11 & 1262 & 1077 & 1136 & \\
\hline 12 & 1263 & 1205 & 1264 & \\
\hline 13 & 1264 & 1309 & 1368 & \\
\hline 14 & 1262 & 1415 & 1474 & \\
\hline \multirow{3}{*}{\multicolumn{4}{|c|}{$\begin{array}{l}\text { Time of the interruption of the refrigerating water } \\
\text { Begining of the measurement of roll surface temperature } \\
\text { Ending of the measurement of roll surface temperature }\end{array}$}} & $1535 \mathrm{sec}$ \\
\hline & & & & $1795 \mathrm{sec}$ \\
\hline & & & & $1975 \mathrm{sec}$ \\
\hline
\end{tabular}

(a) Rolling schedule

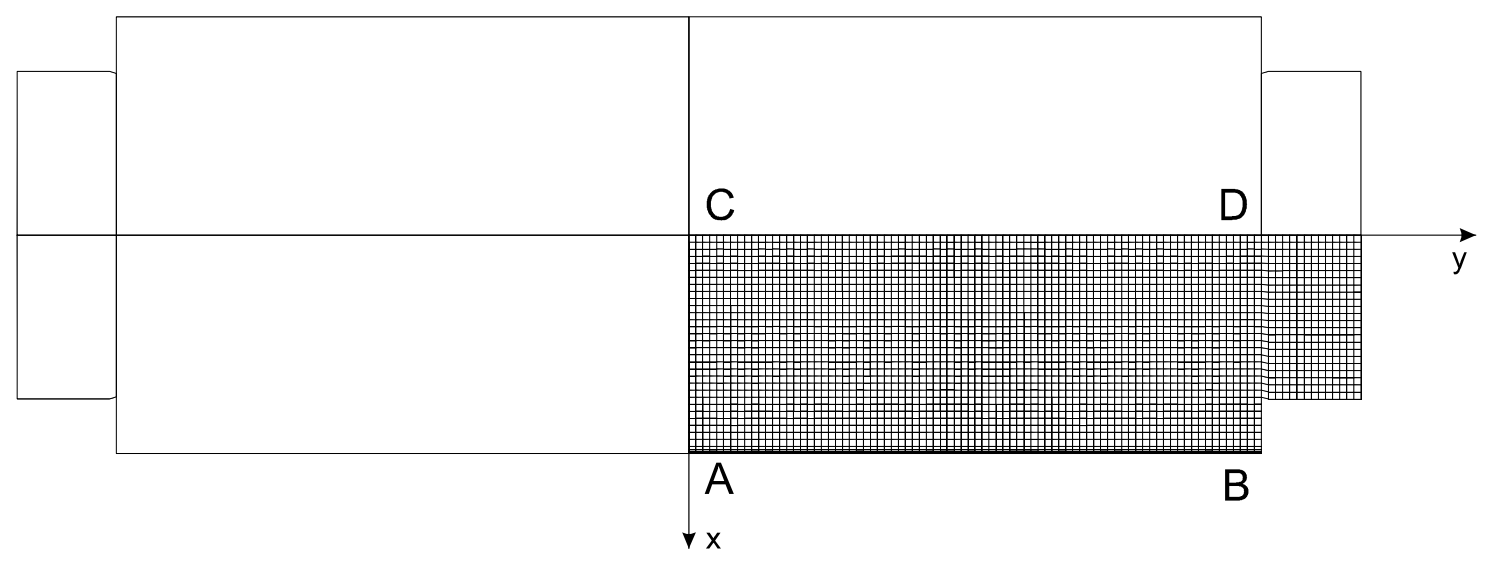

(b) Work roll finite element model (axisymmetric model)

Figure 7. Simulation of the work rolls thermal evolution. 


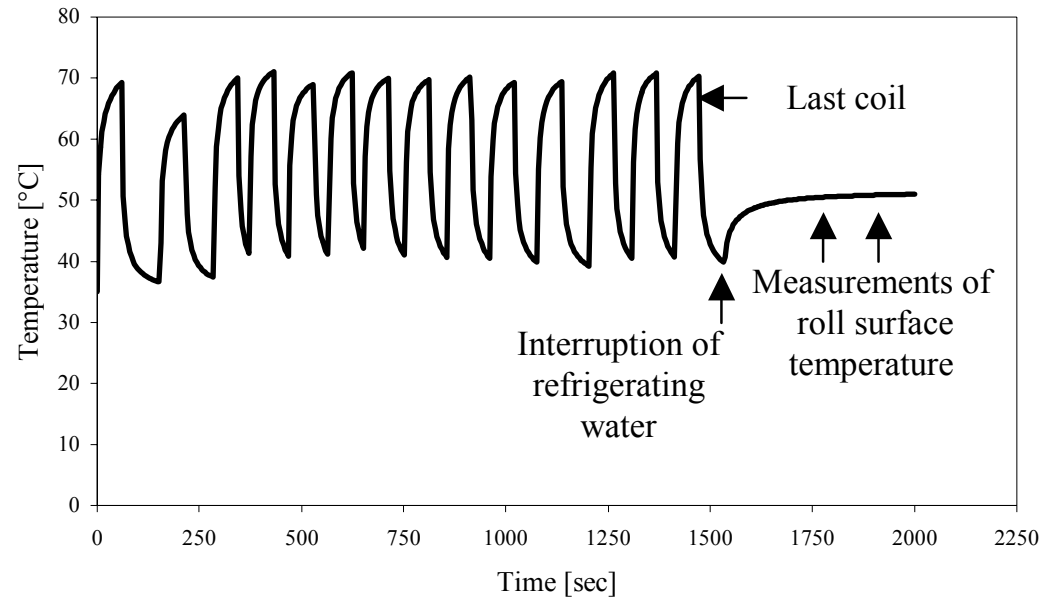

(c) Thermal evolution at point $\mathrm{A}$ (numerical simulation result)

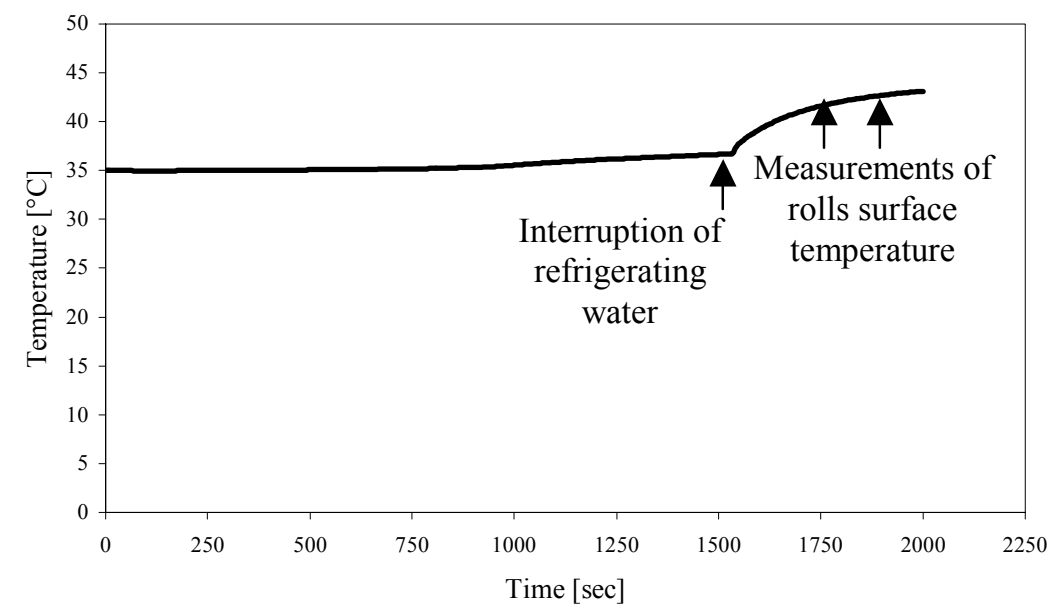

(d) Thermal evolution at point B (numerical simulation result)

Figure 7. (continued) 


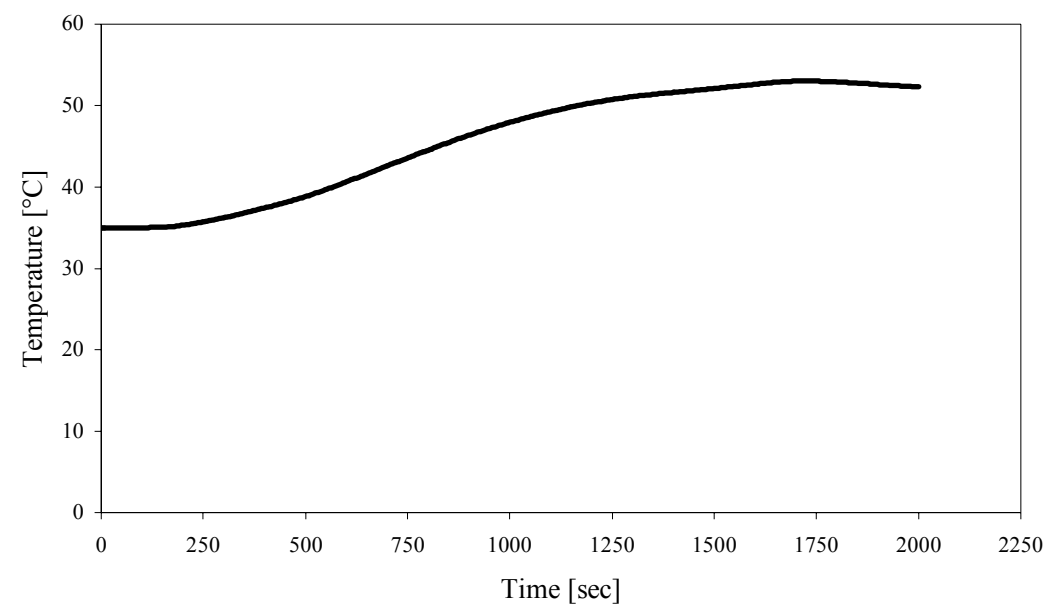

(e) Thermal evolution at point $\mathrm{C}$ (numerical simulation result)

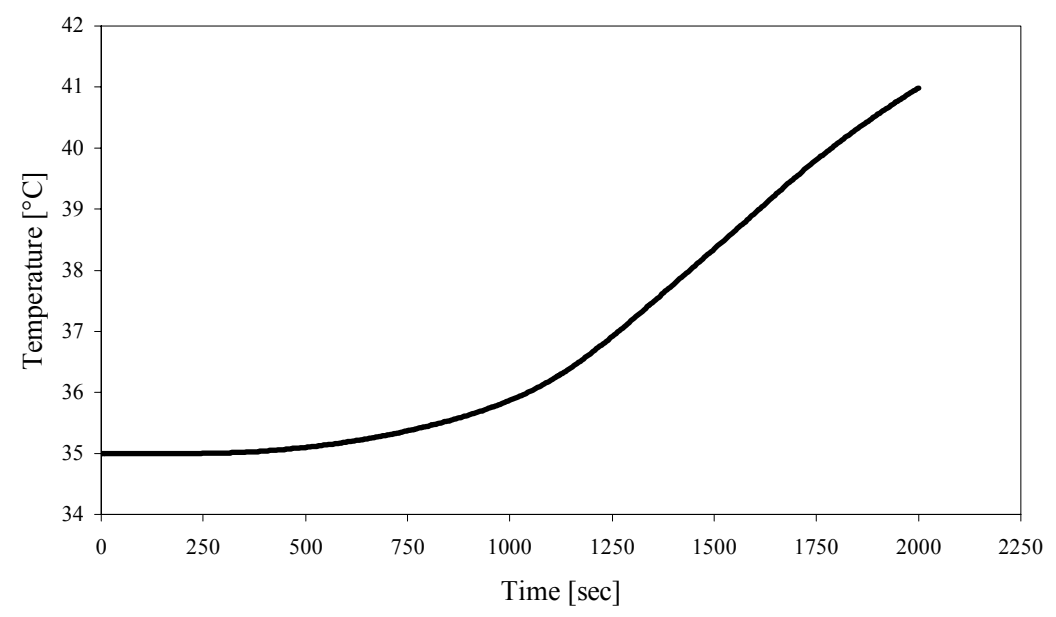

(f) Thermal evolution at point $\mathrm{D}$ (numerical simulation result)

Figure 7. (continued) 


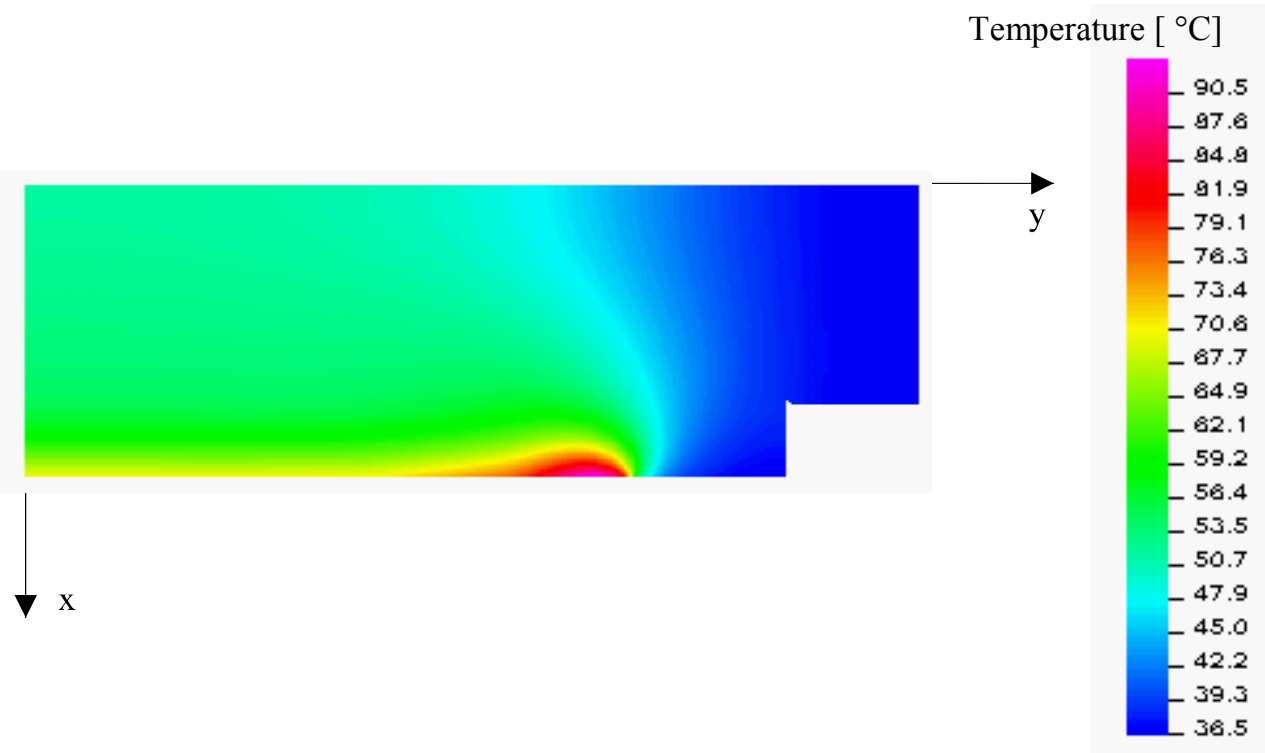

(a) Temperature map of the work roll at the instant at which the last coil exits the stand F10 ( $\left.\mathrm{t}_{\text {out }}\right)$.

Figure 8. Work rolls temperature distribution 


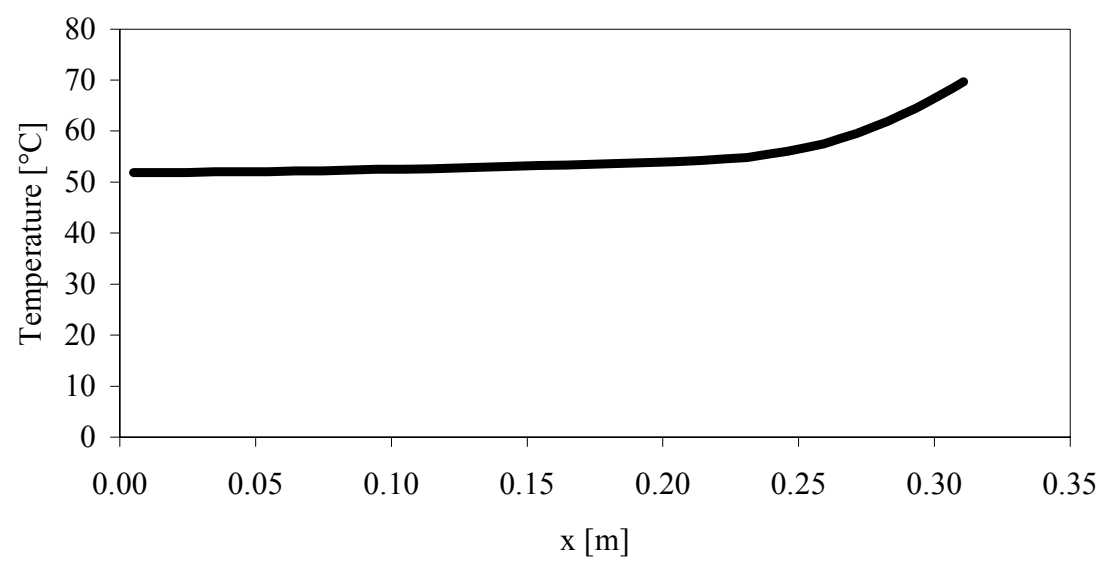

(b) Work roll radial temperature distribution $(\mathrm{y}=0)$ at $\mathrm{t}_{\text {out }}$.

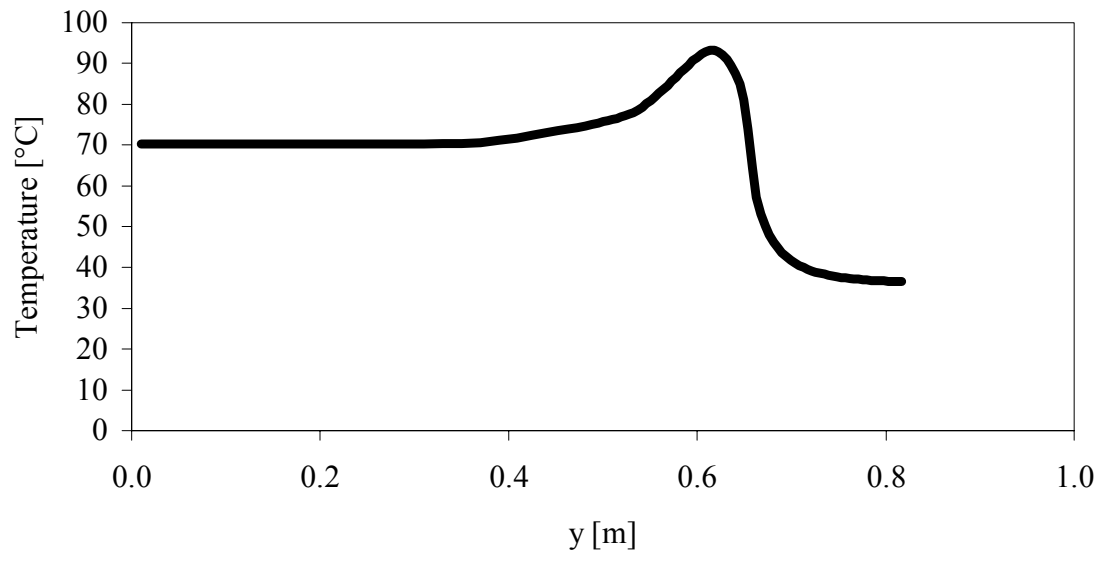

(c) Work roll surface temperature distribution at $t_{\text {out }}$.

Figure 8. (continued) 


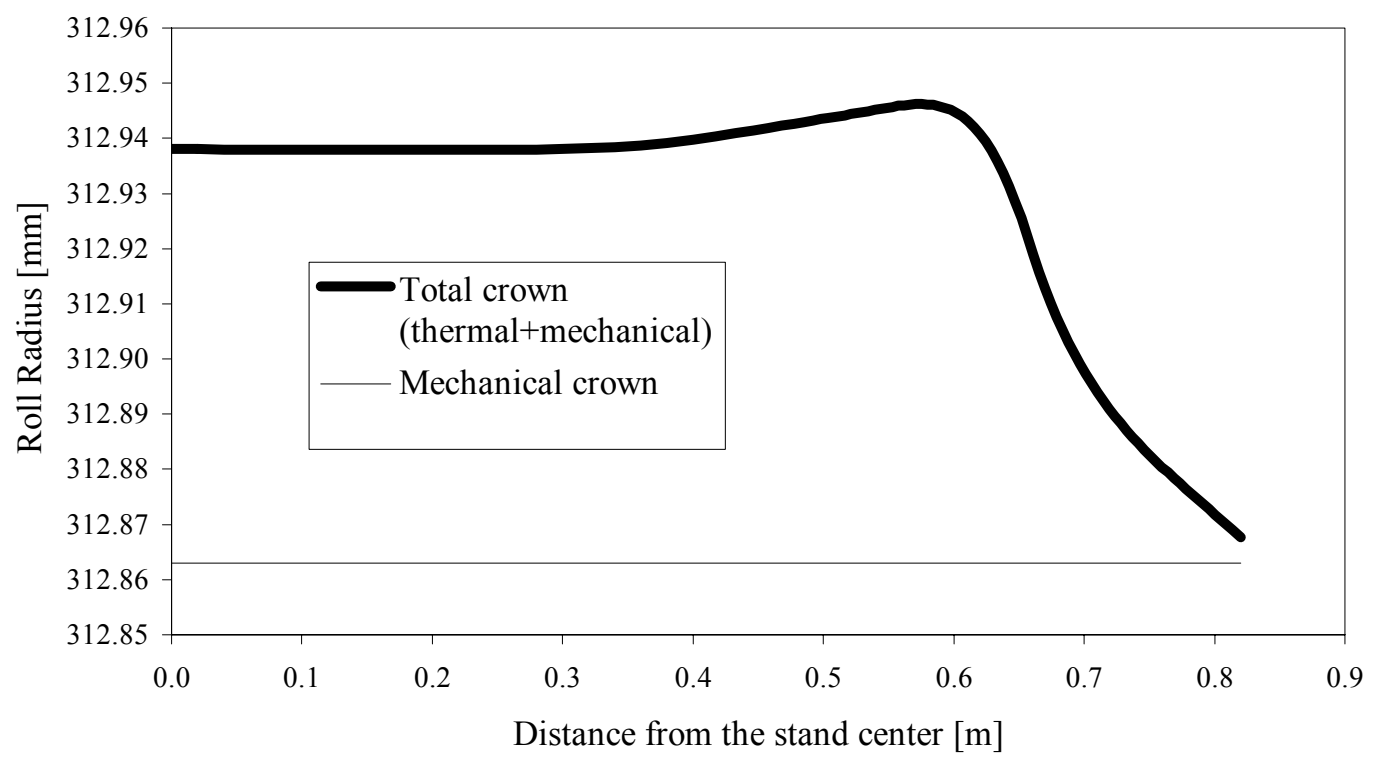

Figure 9. Work roll crown at the F10 stand predicted using TCROWN 


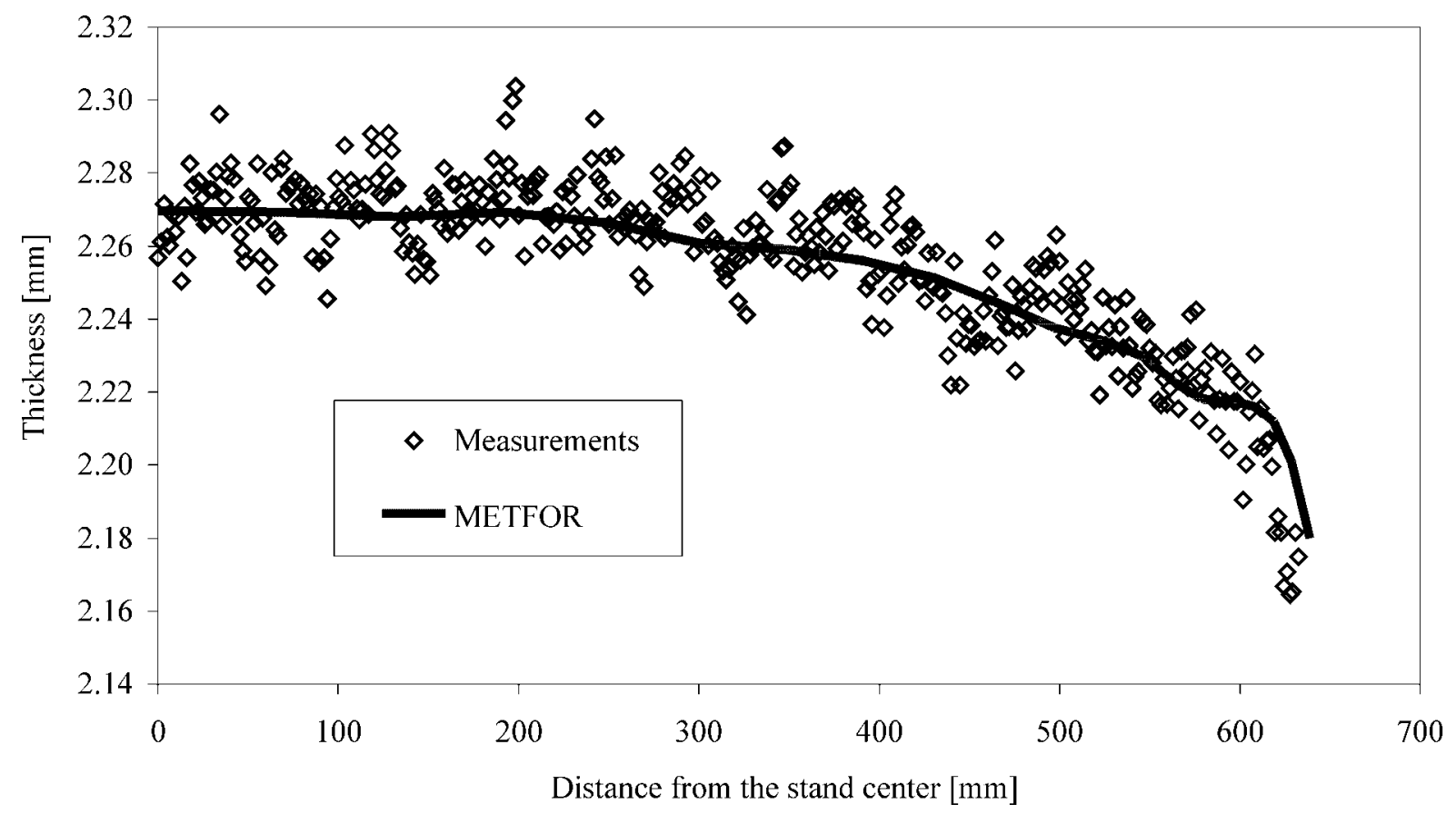

Figure 10. Validation of the finite element results for the F10 stand. 


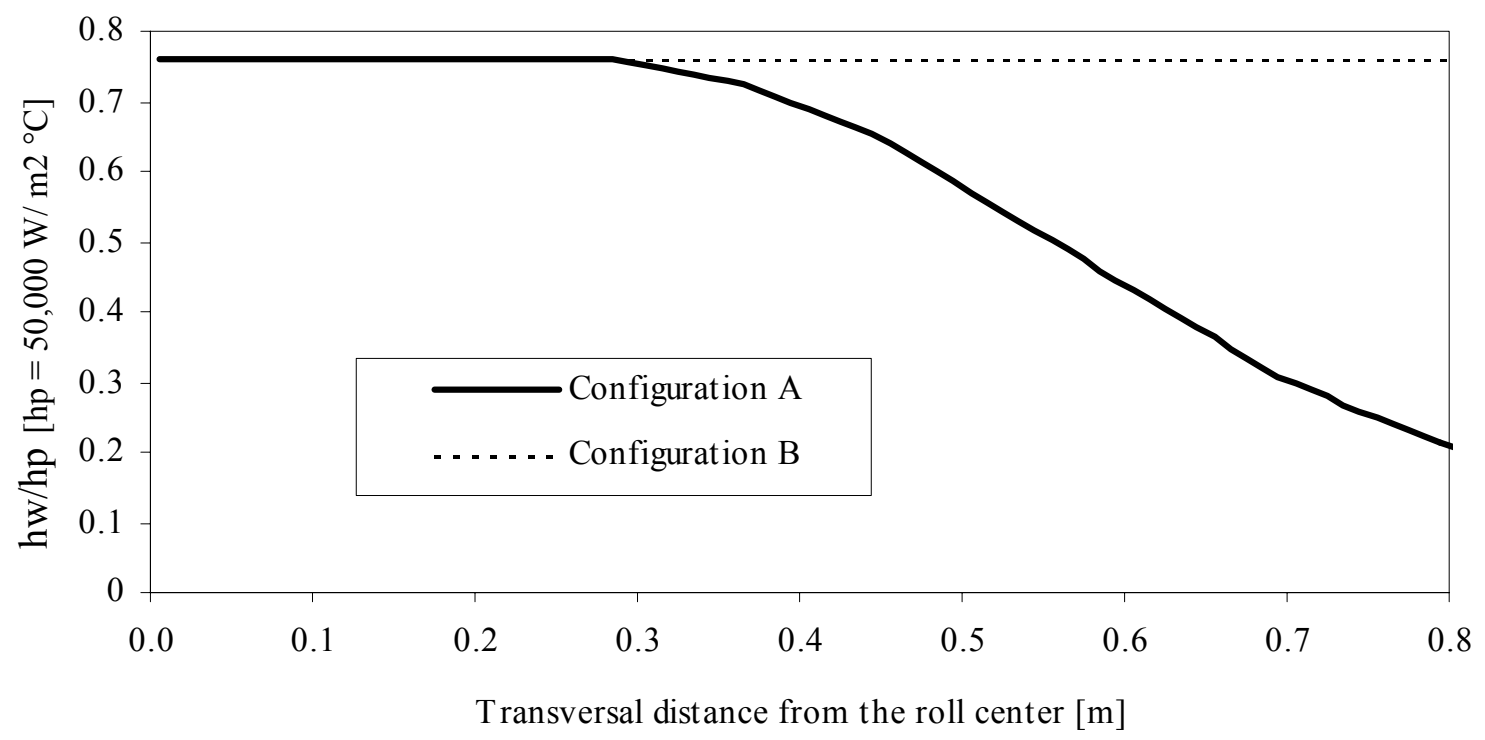

$\mathrm{hp}$ : heat transfer coefficient between plate and roll (assumed constant)

hw : cooling water heat transfer coefficient

Figure 11. Heat transfer capacity for two cooling systems considered at the F10 stand. 


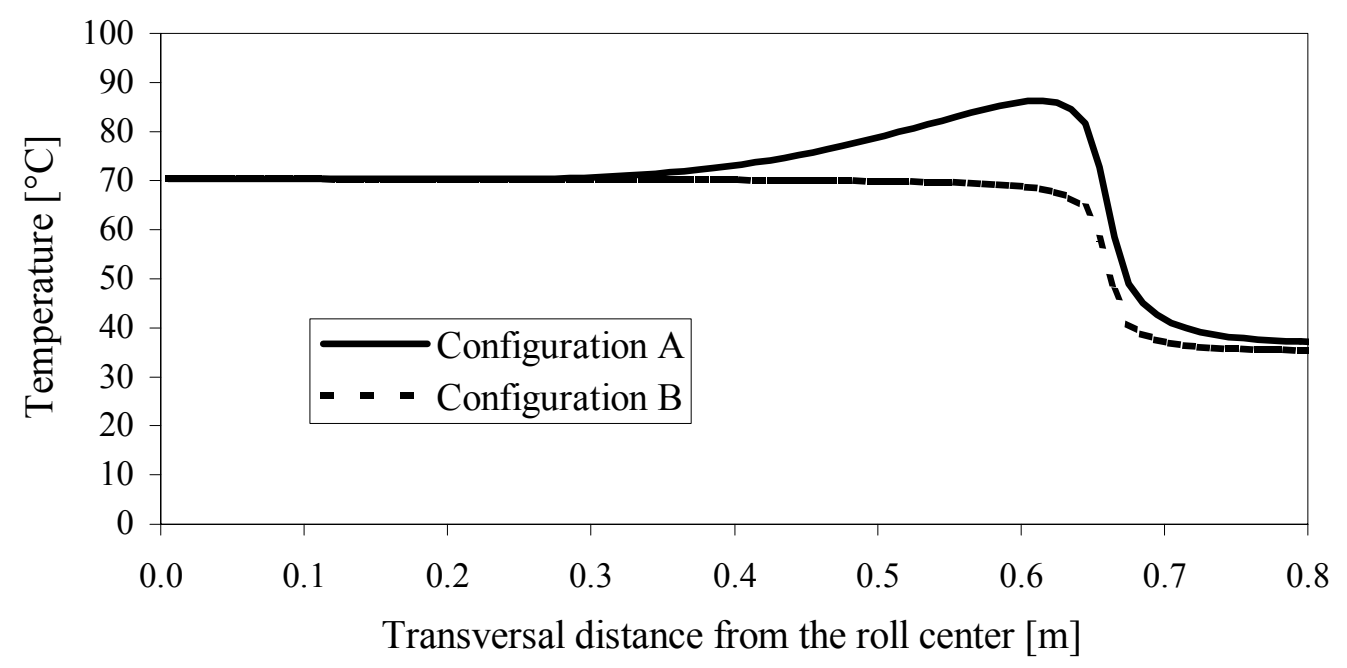

Figure 12. Work roll surface temperature distribution produced by each of the analyzed cooling systems at the F10 stand. 


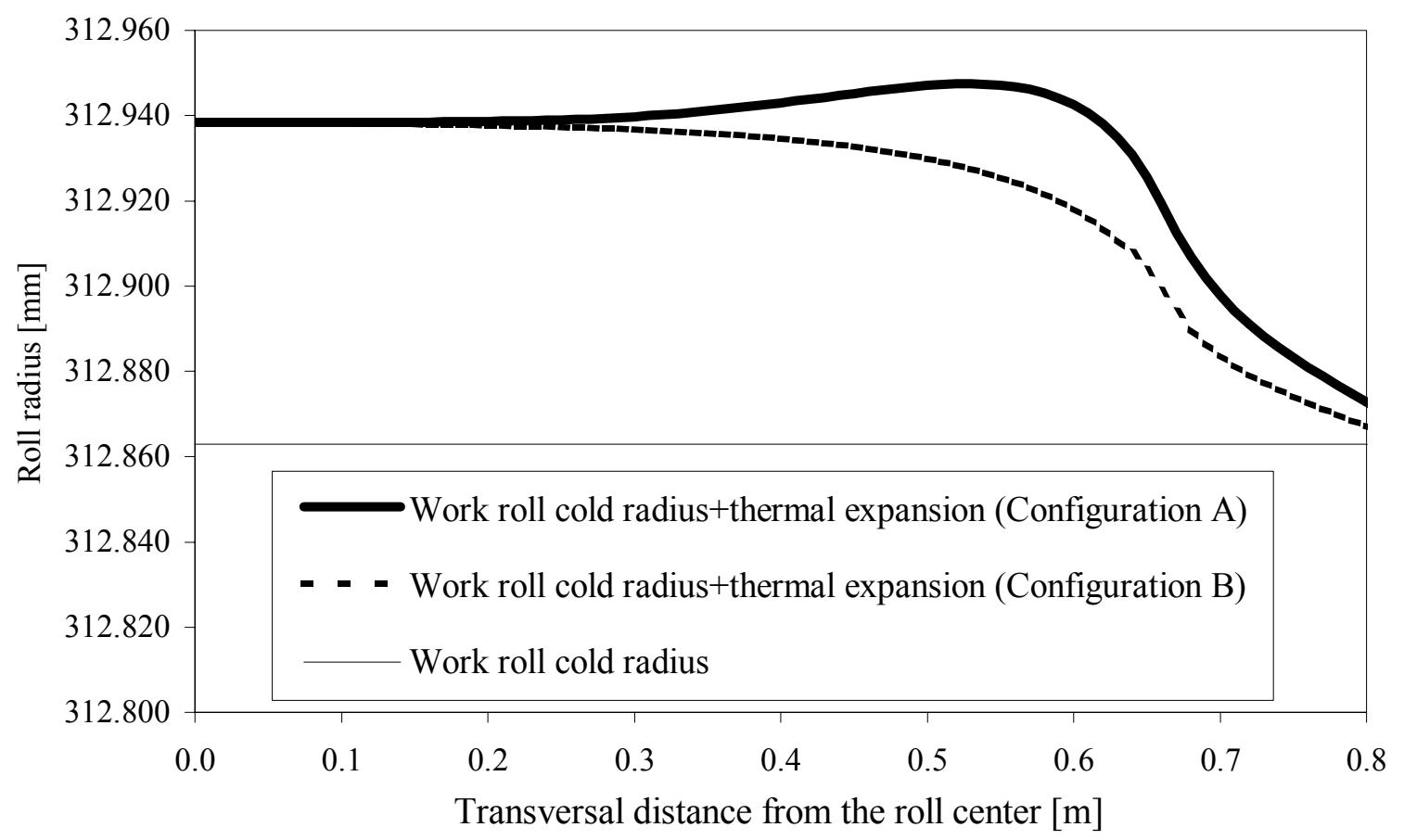

Figure 13. Work roll crowns produced by each of the analyzed cooling systems at the F10 stand. 


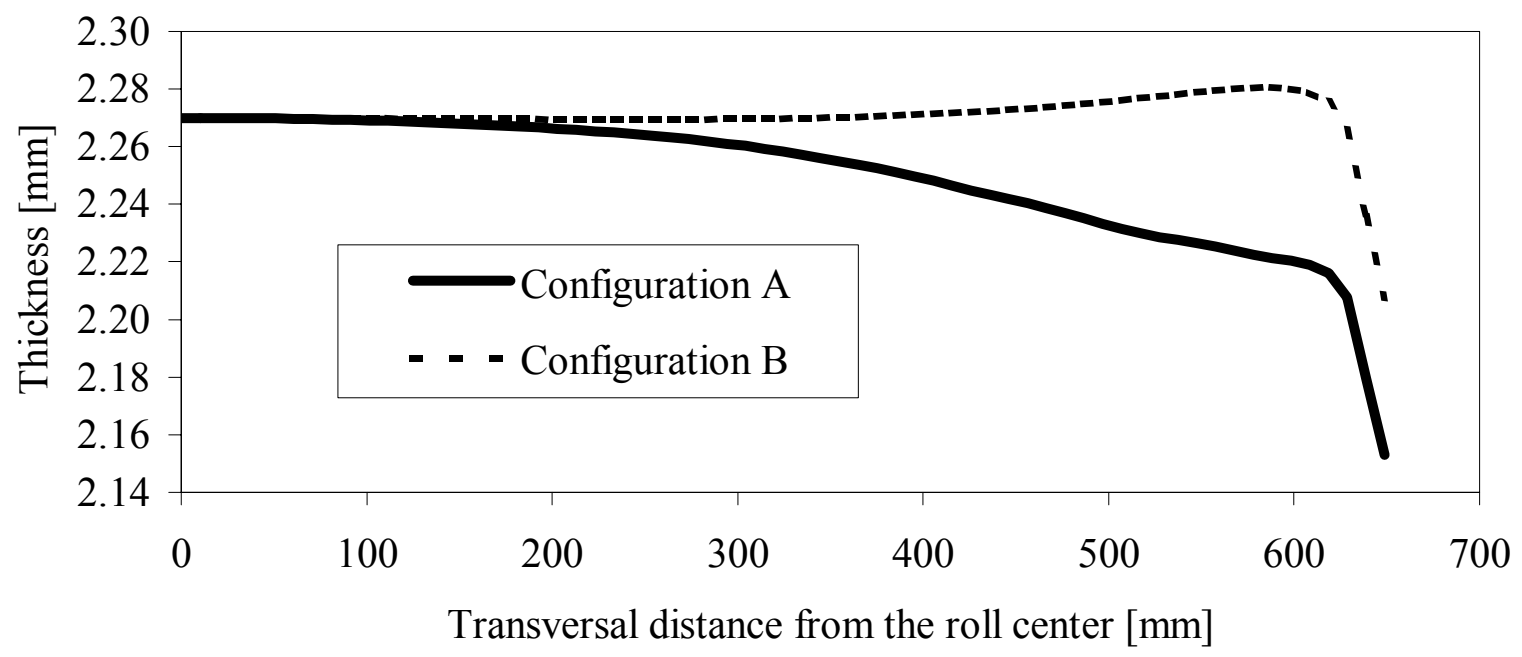

Figure 14. Plate profiles predicted by METFOR for each of the two cooling systems considered 


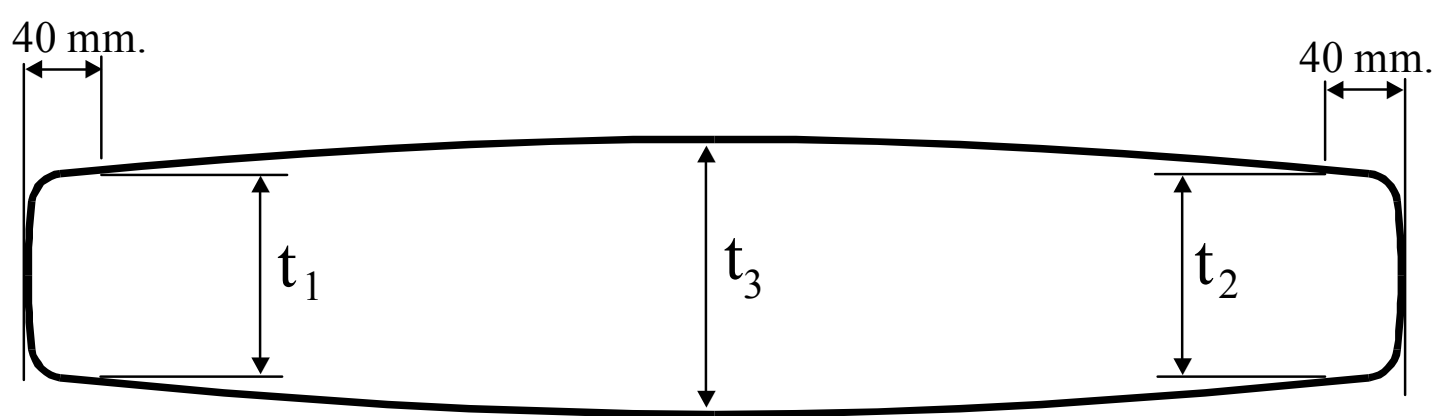

Not to scale

$$
\text { plate crown }=t_{3}-\frac{\left(t_{1}+t_{2}\right)}{2}
$$

Figure A1. Plate profile and crown 


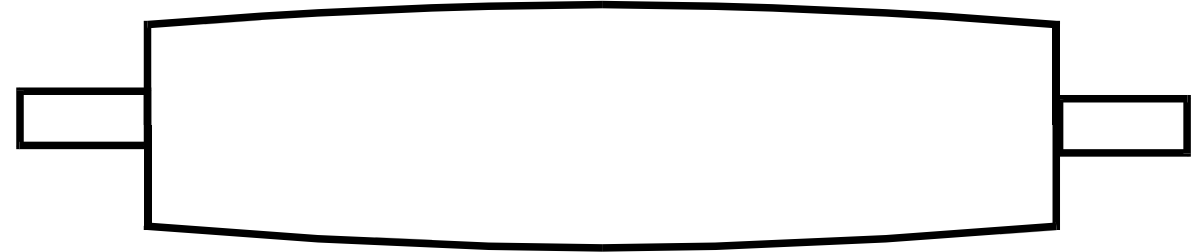

(a) roll with positive crown

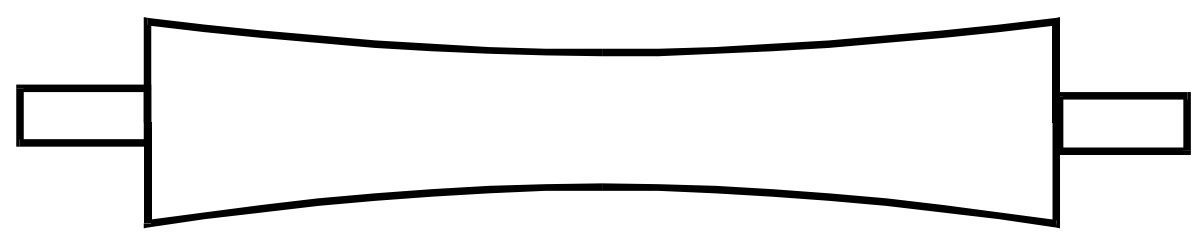

(b) roll with negative crown

roll crown $=$ Center Diameter - Edge Diameter

Figure A2. Rolls profile and crown 